\title{
Common Fixed Point Theorems for Non-compatible Mappings in Intuitionistic Fuzzy Metric space
}

\author{
Saurabh Manro \\ School of Mathematics and Computer Applications, \\ Thapar University, \\ Patiala (Punjab)
}

\begin{abstract}
In this paper, we prove common fixed point theorems for the class of four non compatible mappings in Intuitionistic fuzzy metric spaces are proved. These results are proved without exploiting the notion of continuity and without imposing any condition of $t$-norm and $t$-conorm. Our results generalize several comparable results in existing literature.
\end{abstract}

\section{Keywords}

Intuitionistic fuzzy metric space, E.A. property, Common E.A. property, Weakly compatible maps, Common fixed point.

\section{SUBJECT CLASSIFICATION $47 \mathrm{H} 10,54 \mathrm{H} 25$}

\section{INTRODUCTION}

As a generalization of fuzzy sets introduced by Zadeh [1], Atanassove[2] introduced and studied the concept of intuitionistic fuzzy sets as a generalization of fuzzy sets. In 2004, Park[3] defined the notion of intuitionistic fuzzy metric space with the help of continuous $t$-norms and continuous $t$ conorms. Recently, in 2006, Alaca et al.[4] using the idea of Intuitionistic fuzzy sets, defined the notion of intuitionistic fuzzy metric space with the help of continuous t-norm and continuous t- conorms as a generalization of fuzzy metric space due to Kramosil and Michalek[5]. In 2006, Turkoglu [6] proved Jungck's[7] common fixed point theorem in the in the setting of intuitionistic fuzzy metric spaces for commuting mappings. Afterwards, many authors proved common fixed point theorems using different variants in such spaces. In this paper, we prove common fixed point theorems for the class of four non compatible mappings in Intuitionistic fuzzy metric spaces are proved. These results are proved without exploiting the notion of continuity and without imposing any condition of $t$-norm and $t$-conorm. Our results generalize several comparable results in existing literature.

\section{PRELIMINARIES}

The concepts of triangular norms $(t-$ norm $)$ and triangular conorms $(t$ - conorm) are known as the axiomatic skelton that we use are characterization fuzzy intersections and union respectively. These concepts were originally introduced by Menger in study of statistical metric spaces.

\section{Definition 2.1[8]}

A binary operation $*:[0,1] \times[0,1] \rightarrow[0,1]$ is continuous $t$-norm if $*$ satisfies the following conditions:

(i) $*$ is commutative and associative;

(ii) $*$ is continuous;

(iii) $a * 1=a$ for all $a \in[0,1]$;

(iv) $a * b \leq c * d$ whenever $a \leq c$ and $b \leq d$ for all $a, b, c, d \in[0,1]$.

\section{Definition 2.2[8]}

A binary operation $\diamond:[0,1] \times[0,1] \rightarrow[0,1]$ is continuous $t$-conorm if $\diamond$ satisfies the following conditions:

(i) $\diamond$ is commutative and associative;

(ii) $\diamond$ is continuous;

(iii) $a \diamond 0=a$ for all $a \in[0,1]$;

(iv) $a \diamond b \leq c \diamond d$ whenever $a \leq c$ and $b \leq d$ for all $a, b, c, d \in[0,1]$.

Alaca et al.[4] defined the notion of intuitionistic fuzzy metric space as :

\section{Definition 2.3[4]}

A 5-tuple $(X, M, N, *, \diamond)$ is said to be an intuitionistic fuzzy metric space if $X$ is an arbitrary set, $*$ is a continuous $t$-norm, $\diamond$ is a continuous $t$-conorm and $M, N$ are fuzzy sets on $\mathrm{X}^{2} \times[0, \infty)$ satisfying the conditions:

(i) $M(x, y, t)+N(x, y, t) \leq 1$ for all $x, y \in X$ and $t>0$

(ii) $M(x, y, 0)=0$ for all $x, y \in X$;

(iii) $M(x, y, t)=1$ for all $x, y \in X$ and $t>0$ if and only if $x=y$;

(iv) $M(x, y, t)=M(y, x, t)$ for all $x, y \in X$ and $t>0$;

(v) $M(x, y, t) * M(y, z, s) \leq M(x, z, t+s)$

for all $x, y, z \in X$ and $s, t>0$;

(vi) $M(x, y,):.[0, \infty) \rightarrow[0,1]$ is left continuous, for all $x, y \in X$; 
(vii) $\lim _{t \rightarrow \infty} \mathrm{M}(\mathrm{x}, \mathrm{y}, \mathrm{t})=1$ for all $\quad x, y \in X$ and $t>0$;

(viii) $N(x, y, 0)=1$ for all $x, y \in X$;

(ix) $N(x, y, t)=0$ for all $x, y \in X$ and $t>0$ if and only if $x=y$;

(x) $N(x, y, t)=N(y, x, t)$ for all $x, y \in X$ and $t>0$;

(xi) $N(x, y, t) \diamond N(y, z, s) \geq N(x, z, t+s)$

for all $x, y, z \in X$ and $s, t>0$;

(xii) $N(x, y,):.[0, \infty) \rightarrow[0,1]$ is right continuous, for all $x, y \in X$;

(xiii) $\lim _{t \rightarrow \infty} N(\mathrm{x}, \mathrm{y}, \mathrm{t})=0$ for all $\quad x, y \in X$.

The functions $M(x, y, t)$ and $N(x, y, t)$ denote the degree of nearness and the degree of non-nearness between $x$ and $y$ w.r.t. $t$ respectively.

\section{Remark 2.1[9]}

Every fuzzy metric space $(X, M, *)$ is an intuitionistic fuzzy metric space of the form $(X, M, 1-M, *, \diamond)$ such that $t$-norm $*$ and $t$-conorm $\diamond$ are associated as

$$
x \diamond y=1-((1-x) *(1-y)) \text { for all } x, y \in X \text {. }
$$

\section{Remark 2.2[9]}

In intuitionistic fuzzy metric space $(X, M, N, *, \oslash)$, $M\left(x, y,{ }^{*}\right)$ is non-decreasing and $N(x, y, \diamond)$ is nonincreasing for all $x, y \in X$.

\section{Definition 2.4[4]}

Let $(X, M, N, *, \diamond)$ be an intuitionistic fuzzy metric space. Then

(a) a sequence $\left\{x_{n}\right\}$ in $X$ is said to be Cauchy sequence if, for all $t>0$ and $p>0$,

$$
\begin{aligned}
& \lim _{n \rightarrow \infty} \mathrm{M}\left(\mathrm{x}_{\mathrm{n}+\mathrm{p}}, \mathrm{x}_{\mathrm{n}}, \mathrm{t}\right)=1 \\
& \text { and } \\
& \lim _{n \rightarrow \infty} N\left(\mathrm{x}_{\mathrm{n}+\mathrm{p}}, \mathrm{x}_{\mathrm{n}}, \mathrm{t}\right)=0 .
\end{aligned}
$$

(b) a sequence $\left\{x_{n}\right\}$ in $X$ is said to be convergent to a point $x \in X$ if, for all $t>0$,

$\lim _{n \rightarrow \infty} M\left(x_{n}, x, t\right)=1$

and

$\lim _{n \rightarrow \infty} N\left(x_{n}, x, t\right)=0$.

\section{Definition 2.5[4]}

An intuitionistic fuzzy metric space $(X, M, N, *, \diamond)$ is said to be complete if and only if every Cauchy sequence in $X$ is convergent.

\section{Example 2.1[4]}

Let $X=\left\{\frac{1}{n}: n \in N\right\} \cup\{0\}$ and let $*$ be the continuous $t$-norm and $\diamond$ be the continuous $t$-conorm defined by $a^{*} b=a b$ and $a \diamond b=\min \{1, a+b\}$ respectively,

for all $a, b \in[0,1]$.

For each $t \in(0, \infty)$ and $x, y \in X$, define $\mathrm{M}$ and $\mathrm{N}$ by

$M(x, y, t)=\left\{\begin{array}{cc}\frac{\mathrm{t}}{\mathrm{t}+|\mathrm{X}-\mathrm{y}|}, & \mathrm{t}>\mathrm{O} \\ \mathrm{O}, & \mathrm{t}=\mathrm{O}\end{array}\right.$

and

$N(x, y, t)=\left\{\begin{array}{cc}\frac{|\mathrm{x}-\mathrm{y}|}{\mathrm{t}+|\mathrm{x}-\mathrm{y}|}, & \mathrm{t}>0 \\ 1, & \mathrm{t}=0\end{array}\right.$

Clearly,

$(X, M, N, *, \diamond)$ is complete intuitionistic fuzzy metric space.

\section{Definition 2.6[4]}

A pair of self mappings $(A, S)$ of a intuitionistic fuzzy metric space $(X, M, N, *, \diamond)$ is said to be commuting if $M(A S x, S A x, t)=1$ and $N(A S x, S A x, t)=0$ for all $x \in X$.

\section{Definition 2.7[4]}

A pair of self mappings $(A, S)$ of a intuitionistic fuzzy metric space $(X, M, N, *, \diamond)$ is said to be weakly commuting if

$M(A S x, S A x, t) \geq M(A x, S x, t)$ and

$N(A S x, S A x, t) \leq N(A x, S x, t)$

for all $x \in X$ and $t>0$.

\section{Definition 2.8[9]}

A pair of self mappings $(A, S)$ of a intuitionistic fuzzy metric space $(X, M, N, *, \diamond)$ is said to be compatible if $\lim _{n \rightarrow \infty} M\left(A S x_{n}, S A x_{n}, t\right)=1$, and $\lim _{n \rightarrow \infty} N\left(A S x_{n}, S A x_{n}, t\right)=0$ for all $t>0$, whenever $\left\{x_{n}\right\}$ is a sequence in $X$ such that $\lim _{n \rightarrow \infty} A x_{n}=\lim _{n \rightarrow \infty} S x_{n}=u$ for some $u \in X$.

\section{Definition 2.9[10]}

Let $(X, M, N, *, \diamond)$ be a intuitionistic fuzzy metric space. $A$ and $S$ be self maps on $X$. A point $x \in X$ is called a coincidence point of $A$ and $S$ iff $A x=S x$. In this case, $w=A x$ $=S x$ is called a point of coincidence of $A$ and $S$. 


\section{Definition 2.10[10]}

A pair of self mappings $(A, S)$ of a intuitionistic fuzzy metric space $(X, M, N, *, \diamond)$ is said to be weakly compatible if they commute at the coincidence points i.e., if $\mathrm{Au}=\mathrm{Su}$ for some, then $\mathrm{ASu}=\mathrm{SAu}$.

It is easy to see that two compatible maps are weakly compatible but converse is not true.

It was pointed out by Aamri and Moutawakil[11] that E.A. property buys containment of ranges without any continuity requirements, besides minimize the commutativity conditions of the maps to the commutativity at their points of coincidence. Moreover E.A. property allows replacing the completeness requirement of the space with a more natural condition of closeness of the range. Some common fixed point theorems in probabilistic or fuzzy metric spaces by E.A. property under weak compatibility have been recently obtained by many authors. The aim of this paper is to strengthen these results and to emphasize the role of E.A. property in the existence of common fixed point.

Aamri and Moutawakil [11] introduced the notion of E.A. property in metric spaces as follows:

\section{Definition 2.12[11]}

A pair of self mappings $(A, S)$ on an intuitionistic fuzzy metric space $(X, M, N, *, \diamond)$ is said to satisfy the E.A. property if there exist a sequence $\left\{x_{n}\right\}$ in $X$ such that

$$
\lim _{n \rightarrow \infty} A x_{n}=z=\lim _{n \rightarrow \infty} S x_{n} \text { for some } z \in X .
$$

\section{Definition 2.13[10]}

Mappings $A, B, S$ and $T$ on an intuitionistic fuzzy metric space $(X, M, N, *, \diamond)$ are said to satisfy the common E.A. property if there exist a sequences

$\left\{x_{n}\right\}$ and $\left\{y_{n}\right\}$ in $X$ such that

$\lim _{n \rightarrow \infty} B y_{n}=\lim _{n \rightarrow \infty} T y_{n}=\lim _{n \rightarrow \infty} A x_{n}=\lim _{n \rightarrow \infty} S x_{n}=z \quad$ for

some $z \in X$

For more on E.A. and common E.A. properties, we refer to [11] and [10].

\section{LEMMAS}

The proof of our result is based upon the following lemmas of which the first two are due to Alaca et al.[4]:

\section{Lemma 3.1[4]}

Let $(X, M, N, *, \diamond)$ be intuitionistic fuzzy metric space and for all $x, y \in X, t>0$ and if for a number $k \in(0,1)$,

$M(x, y, k t) \geq M(x, y, t)$ and $N(x, y, k t) \leq N(x, y, t)$

Then $\mathrm{x}=\mathrm{y}$.

\section{Lemma 3.2[4]}

Let $\left\{u_{n}\right\}$ is a sequence in a intuitionistic fuzzy metric space $(X, M, N, *, \oslash)$. If there exists a constant $k \in(0,1)$ such that

$M\left(u_{n}, u_{n+1}, k t\right) \geq M\left(u_{n-1}, u_{n}, t\right)$,

$N\left(u_{n}, u_{n+1}, k t\right) \leq N\left(u_{n-1}, u_{n}, t\right)$

for all $n=0,1,2, \ldots$

Then $\left\{u_{n}\right\}$ is a Cauchy sequence in $X$.

\section{MAIN RESULT}

Let $\Psi, \Phi$ a class of implicit relations be the set of all continuous functions $\psi \in \Psi, \phi \in \Phi$ such that $\psi:[0,1]^{5} \rightarrow[0,1]$, $\phi:[0,1]^{5} \rightarrow[0,1]$ which are increasing in each coordinate and $\psi(t, t, t, t, t)<t, \phi(t, t, t, t, t)>t$ for all $t \in[0,1)$.

\section{Theorem 4.1}

Let $(X, M, N, *, \diamond)$ be a intuitionistic fuzzy metric space. Let $A, B, S$ and $T$ be maps from $X$ into itself satisfying:

(4.1) there exists a constant $k \in\left(0, \frac{1}{2}\right)$ such that

$M(A x, B y, k t) \geq \phi\left(\begin{array}{l}M(S x, T y, t), M(A x, S x, t), \\ M(B y, T y, t), M(A x, T y, \alpha t), M(B y, S x,(2-\alpha) t)\end{array}\right)$ for all

$N(A x, B y, k t) \leq \psi\left(\begin{array}{l}N(S x, T y, t), N(A x, S x, t), \\ N(B y, T y, t), N(A x, T y, \alpha t), N(B y, S x,(2-\alpha) t)\end{array}\right)$

$x, y \in X, \alpha \in(0,2), t>0$ and $\phi \in \Phi$,

(4.2) $A(X) \subseteq T(X), B(X) \subseteq S(X)$,

(4.3) the pair $\{A, S\}$ or $\{B, T\}$ satisfies E.A. property,

If one of $A(X), T(X), S(X)$ or $B(X)$ is closed subset of $X$ and the pairs $\{A, S\}$ and $\{B, T\}$ are weakly compatible then $A, B, S$ and $T$ have a unique common fixed point in $X$.

\section{Proof}

Suppose that a pair $\{B, T\}$ satisfies E.A. property, therefore there exists a sequence $\left\{x_{n}\right\}$ in $X$ such that $\lim _{n \rightarrow \infty} B x_{n}=z=\lim _{n \rightarrow \infty} T x_{n} . \quad$ Now, $\quad B(X) \subseteq S(X)$ implies that there exists a sequence $\left\{y_{n}\right\}$ in $X$ such that $B x_{n}=$ $S y_{n}$. For $\alpha=1, x=y_{n}, y=x_{n}$ in (4.1), we get

$$
\begin{aligned}
& M\left(A y_{n}, B x_{n}, k t\right) \geq \phi\left(\begin{array}{l}
M\left(S y_{n}, T x_{n}, t\right), M\left(A y_{n}, S y_{n}, t\right), \\
M\left(B x_{n}, T x_{n}, t\right), M\left(A y_{n}, T x_{n}, t\right), M\left(B x_{n}, S y_{n}, t\right)
\end{array}\right) \\
& N\left(A y_{n}, B x_{n}, k t\right) \leq \psi\left(\begin{array}{l}
N\left(S y_{n}, T x_{n}, t\right), N\left(A y_{n}, S y_{n}, t\right), \\
N\left(B x_{n}, T x_{n}, t\right), N\left(A y_{n}, T x_{n}, t\right), N\left(B x_{n}, S y_{n}, t\right)
\end{array}\right) \\
& n \rightarrow \infty
\end{aligned}
$$




$$
\begin{aligned}
& M\left(\lim _{n \rightarrow \infty} A y_{n}, z, k t\right) \geq \phi\left(\begin{array}{l}
M(z, z, t), M\left(\lim _{n \rightarrow \infty} A y_{n}, z, t\right), \\
M(z, z, t), M\left(\lim _{n \rightarrow \infty} A y_{n}, z, t\right), M(z, z, t)
\end{array}\right) \text { As } \\
& N\left(\lim _{n \rightarrow \infty} A y_{n}, z, k t\right) \leq \psi\left(\begin{array}{l}
N(z, z, t), N\left(\lim _{n \rightarrow \infty} A y_{n}, z, t\right), \\
N(z, z, t), N\left(\lim _{n \rightarrow \infty} A y_{n}, z, t\right), N(z, z, t)
\end{array}\right) \\
& \phi, \psi \quad \text { are increasing in each of its ordinate and } \\
& \phi(t, t, t, t, t)>t, \psi(t, t, t, t, t)<t \text { for all } t \in[0,1), \\
& M\left(\lim _{n \rightarrow \infty} A y_{n}, z, k t\right)>M\left(\lim _{n \rightarrow \infty} A y_{n}, z, k t\right), \\
& N\left(\lim _{n \rightarrow \infty} A y_{n}, z, k t\right)<N\left(\lim _{n \rightarrow \infty} A y_{n}, z, k t\right)
\end{aligned}
$$

which by lemma 3.1, implies that $\lim _{n \rightarrow \infty} A y_{n}=z$. Suppose that $S(X)$ is a closed subspace of $X$. Then, $z=S u$ for some $u \in X$. Now, take $x=u, y=x_{n}, \alpha=1$ in (4.1), we have

$$
\begin{aligned}
& M\left(A u, B x_{n}, k t\right) \geq \phi\left(\begin{array}{l}
M\left(S u, T x_{n}, t\right), M(A u, S u, t), \\
M\left(B x_{n}, T x_{n}, t\right), M\left(A u, T x_{n}, \alpha t\right), M\left(B x_{n}, S u,(2-\alpha) t\right)
\end{array}\right) \\
& N\left(A u, B x_{n}, k t\right) \leq \psi\left(\begin{array}{l}
N\left(S u, T x_{n}, t\right), N(A u, S u, t), \\
N\left(B x_{n}, T x_{n}, t\right), N\left(A u, T x_{n}, \alpha t\right), N\left(B x_{n}, S u,(2-\alpha) t\right)
\end{array}\right) \\
& n \rightarrow \infty \\
& M(A u, z, k t) \geq \phi\left(\begin{array}{l}
M(z, z, t), M(A u, z, t), \\
M(z, z, t), M(A u, z, t), M(z, z, t)
\end{array}\right) \\
& N(A u, z, k t) \leq \psi\left(\begin{array}{l}
N(z, z, t), N(A u, z, t), \\
N(z, z, t), N(A u, z, t), N(z, z, t)
\end{array}\right) \\
& \Rightarrow M(A u, z, k t) \geq M(A u, z, t), \\
& N(A u, z, k t) \leq N(A u, z, t)
\end{aligned}
$$

which by lemma 3.1, implies that $A u=z$. Hence, $A u=z=S u$. Since $A(X) \subseteq T(X)$, there exists $v \in X$ such that $T v=$ $z$. Following the arguments similar to those given above, we obtain $z=B v=T v$. Since $u$ is a coincidence point of pair $\{A, S\}$ therefore, $A S u=S A u$ and $A z=S z$. Now we claim that $A z=z$, if not, then using (4.1), take $\alpha=1, x=z, y=v$, we have $M(A z, B v, k t) \geq \phi\left(\begin{array}{l}M(S z, T v, t), M(A z, S z, t), \\ M(B v, T v, t), M(A z, T v, t), M(B v, S z, t)\end{array}\right)$ $N(A z, B v, k t) \leq \psi\left(\begin{array}{l}N(S z, T v, t), N(A z, S z, t), \\ N(B v, T v, t), N(A z, T v, t), N(B v, S z, t)\end{array}\right)$ $M(A z, z, k t) \geq \phi\left(\begin{array}{l}M(A z, z, t), M(A z, A z, t), \\ M(B v, B v, t), M(A z, z, t), M(z, A z, t)\end{array}\right)$ $N(A z, z, k t) \leq \psi\left(\begin{array}{l}N(A z, z, t), N(A z, A z, t), \\ N(B v, B v, t), N(A z, z, t), N(z, A z, t)\end{array}\right)$ $\Rightarrow M(A z, z, k t) \geq M(A z, z, t), N(A z, z, k t) \leq N(A z, z, t)$

Hence by using lemma 3.1, $A z=z=S z$. Similarly, we prove that $z=B z=T z$. This shows that $z$ is common fixed point of $A, B, S$ and $T$.

For uniqueness, let w be another fixed point of $A, B, S$ and $T$. Take $\alpha=1, x=z, y=w$ in (4.1), we have

$$
\begin{aligned}
& M(A z, B w, k t) \geq \phi\left(\begin{array}{l}
M(S z, T w, t), M(A z, S z, t), \\
M(B w, T w, t), M(A z, T w, t), M(B w, S z, t)
\end{array}\right) \\
& N(A z, B w, k t) \leq \psi\left(\begin{array}{l}
N(S z, T w, t), N(A z, S z, t), \\
N(B w, T w, t), N(A z, T w, t), N(B w, S z, t)
\end{array}\right)
\end{aligned}
$$

$$
\begin{aligned}
& M(z, w, k t) \geq \phi\left(\begin{array}{l}
M(z, w, t), M(z, z, t), \\
M(w, w, t), M(z, w, t), M(w, z, t)
\end{array}\right) \\
& N(z, w, k t) \leq \psi\left(\begin{array}{l}
N(z, w, t), N(z, z, t), \\
N(w, w, t), N(z, w, t), N(w, z, t)
\end{array}\right) \text { And by } \\
& \Rightarrow M(z, w, k t) \geq M(z, w, t), N(z, w, k t) \leq N(z, w, t) \\
& \text { using lemma 3.1, we have } z=w . \text { Hence, uniqueness follows. }
\end{aligned}
$$

In our next theorem we prove common fixed point theorem for mappings satisfying common E.A. property.

\section{Theorem 4.2}

Let $(X, M, N, *, \diamond)$ be a intuitionistic fuzzy metric space. Let $A, B, S$ and $T$ be maps from $X$ into itself satisfying (4.2) and:

(4.4) there exists a constant $k \in\left(0, \frac{1}{2}\right)$ such that

$M(A x, B y, k t) \geq \phi\left(\begin{array}{l}M(S x, T y, t), M(A x, S x, t), \\ M(B y, T y, t), M(A x, T y, \alpha t), M(B y, S x,(2-\alpha) t)\end{array}\right)$ for all

$N(A x, B y, k t) \leq \psi\left(\begin{array}{l}N(S x, T y, t), N(A x, S x, t), \\ N(B y, T y, t), N(A x, T y, \alpha t), N(B y, S x,(2-\alpha) t)\end{array}\right)$

$x, y \in X, \alpha \in(0,2), t>0$ and $\phi \in \Phi$,

(4.5) the pairs $\{A, S\}$ and $\{B, T\}$ satisfies common E.A. property,

If one of $A(X), T(X), S(X)$ or $B(X)$ is closed subset of $X$ and the pairs $\{A, S\}$ and $\{B, T\}$ are weakly compatible then $A, B, S$ and $T$ have a unique common fixed point in $X$.

\section{Proof}

Given that the pairs $\{A, S\}$ and $\{B, T\}$ satisfies common E.A. property, therefore there exists two sequences $\left\{x_{n}\right\}$ and $\left\{y_{n}\right\}$ in $X$ such that $\lim _{n \rightarrow \infty} B y_{n}=\lim _{n \rightarrow \infty} T y_{n}=\lim _{n \rightarrow \infty} A x_{n}=\lim _{n \rightarrow \infty} S x_{n}=z \quad$ for some $z \in X$. Since $S(X)$ and $T(X)$ are closed subspace of $X$, therefore, $z=S u=T v$ for some $u, v \in X$. Now, we claim that $A u=z$. For this take $\alpha=1, x=u, y=y_{n}$, in (4.4) we have

$$
\begin{aligned}
& M\left(A u, B y_{n}, k t\right) \geq \phi\left(\begin{array}{l}
M\left(S u, T y_{n}, t\right), M(A u, S u, t), \\
M\left(B y_{n}, T y_{n}, t\right), M\left(A u, T y_{n}, t\right), M\left(B y_{n}, S u, t\right)
\end{array}\right) \\
& N\left(A u, B y_{n}, k t\right) \leq \psi\left(\begin{array}{l}
N\left(S u, T y_{n}, t\right), N(A u, S u, t), \\
N\left(B y_{n}, T y_{n}, t\right), N\left(A u, T y_{n}, t\right), N\left(B y_{n}, S u, t\right)
\end{array}\right) \\
& n \rightarrow \infty \\
& M(A u, z, k t) \geq \phi\left(\begin{array}{l}
M(z, z, t), M(A u, z, t), \\
M(z, z, t), M(A u, z, t), M(z, z, t)
\end{array}\right) \\
& N(A u, z, k t) \leq \psi\left(\begin{array}{l}
N(z, z, t), N(A u, z, t), \\
N(z, z, t), N(A u, z, t), N(z, z, t)
\end{array}\right) \\
& \Rightarrow M(A u, z, k t) \geq M(A u, z, t), N(A u, z, k t) \leq N(A u, z, t) \\
& \text { lemma 3.1,Au=z=Su. }
\end{aligned}
$$

Again using (4.4), take $\alpha=1, x=u, y=v$, we get

$$
\begin{aligned}
& M(A u, B v, k t) \geq \phi\left(\begin{array}{l}
M(S u, T v, t), M(A u, S u, t), \\
M(B v, T v, t), M(A u, T v, t), M(B v, S u, t)
\end{array}\right) \\
& N(A u, B v, k t) \leq \psi\left(\begin{array}{l}
N(S u, T v, t), N(A u, S u, t), \\
N(B v, T v, t), N(A u, T v, t), N(B v, S u, t)
\end{array}\right)
\end{aligned}
$$


$M(T v, B v, k t) \geq \phi\left(\begin{array}{l}M(S u, S u, t), M(S u, S u, t), \\ M(B v, T v, t), M(T v, T v, t), M(B v, T v, t)\end{array}\right)$

$N(T v, B v, k t) \leq \psi\left(\begin{array}{l}N(S u, S u, t), N(S u, S u, t), \\ N(B v, T v, t), N(T v, T v, t), N(B v, T v, t)\end{array}\right)$

$\Rightarrow M(T v, B v, k t) \geq M(T v, B v, t), N(T v, B v, k t) \leq N(T v, B v, t)$

lemma 3.1, we get $T v=B v$ and hence $A u=z=S u=T v=B v$.

The rest of the proof follows as in theorem 4.1 .

\section{ACKNOWLEDGEMENT}

We would like to thank the referee for the critical comments and suggestions for the improvement of my paper.

\section{REFERENCES}

[1] L. A. Zadeh, "Fuzzy sets", Inform. Acad Control, 8(1965), 338-353.

[2] K. Atanassov, "Intuitionistic Fuzzy sets", Fuzzy sets and system, 20(1986), 87-96.

[3] J. H. Park, "Intuitionistic fuzzy metric spaces", Chaos, Solitons \& Fractals, 22(2004), 1039-1046.

[4] C. Alaca, D. Turkoglu, and C. Yildiz, "Fixed points in Intuitionistic fuzzy metric spaces", Chaos, Solitons \& Fractals, 29(2006), 1073-1078.
[5] I. Kramosil and J. Michalek, "Fuzzy metric and Statistical metric spaces", Kybernetica, 11(1975), 326-334.

[6] D. Turkoglu, C. Alaca, Y.J. Cho and C. Yildiz, "Common fixed point theorems in intuitionistic fuzzy metric spaces", Journal of Applied Mathematics and Computing, 22(1-2)(2006), 411-424.

[7] G. Jungck, "Commuting mappings and fixed points", American Mathematical Monthly, 83(1976), 261-263.

[8] B. Schweizer and A. Sklar, "Probabilistic Metric Spaces", North Holland Series in Probability and Applied Mathematics. New York-Amsterdam- Oxford: NorthHolland, XVI, 1983,54-77.

[9] C. Alaca, I. Altun and D. Turkoglu, "On compatible mappings of type (I) and (II) in intuitionistic fuzzy metric spaces". Communications of the Korean Mathematical Society, 23(3)(2008), 427-446.

[10] S. Manro, S. S. Bhatia and S. Kumar, "Common Fixed Point Theorems for Weakly Compatible Maps Satisfying Common (E.A.) Property in Intuitionistic Fuzzy Metric Spaces Using Implicit Relation" Journal of Advanced Studies in Topology, 3(2)(2012), 38-44.

[11] M. Aamri, D. El Moutawakil, "Some new common fixed point theorems under strict contractive conditions", $J$. Math. Anal. Appl., 27(2002)181-188. 